\title{
The Scandinavian Small-for-Gestational Age (SGA) pregnancy and birth cohort - A source to continual insight into fetal growth restriction and long term physical and neurodevelopmental health in mother and offspring
}

\author{
Eli Kjøbli ${ }^{1, *}$, Ragnhild Bach ${ }^{1, *}$, Haakon Skogseth ${ }^{2,3}$ and Geir W. Jacobsen ${ }^{4}$ \\ 1) Department of Technology, NTNU, Trondheim, Norway \\ 2) Clinic of Laboratory Medicine, Trondheim University Hospital, Norway \\ 3) Department of Laboratory Medicine, Women's and Children's Health, NTNU, Trondheim, Norway \\ 4) Department of Public Health and General Practice, NTNU, Trondheim, Norway \\ * Eli Kjøbli and Ragnhild Bach are equally first authors \\ Correspondence: Eli Kjøbli, Institutt for bioingeniørfag, FT, NTNU, E.C. Dahls gate 2, 7002 Trondheim \\ E-mail: Eli.Kjobli@ntnu.no Telephone:+47 73412195/+4740461053
}

\begin{abstract}
Human in utero growth restriction (IUGR) is associated with an increased risk for perinatal mortality and morbidity among newborns and infants. To pursue this challenge, a Request For Proposals (RFP) was issued in 1983 by The U.S. Epidemiology and Biometry Research Program at the National Institute of Child Health and Human Development (NICHD). A consortium was set up at the universities and university hospitals in Trondheim, Bergen (Norway) and Uppsala (Sweden) and was funded by the NICHD to conduct the Scandinavian Successive Small-for-Gestational Age (SGA) Pregnancy and Birth Outcome Study. The study design included a comprehensive biobank with maternal and cord serum samples, placental tissue, and a multitude of data collected from interviews, questionnaires, and clinical examinations.

The SGA cohort study involved 6,354 Caucasian pregnant women in the three study sites who expected their second or third child from 1986-88. The study women were screened in early second trimester and mothers who had an increased risk to deliver a smaller than expected newborn were followed in detail through the second half of pregnancy and at birth. Selected children were screened several times through their first and up to five years of age. Moreover, a highly selected subgroup in Trondheim has been followed at 14, 19, and 26 years' age.

Almost thirty years later, we have searched the body of scientific publications that originated from this cohort study in an attempt to assess if and to what extent the main aims and objectives were achieved and to summarize the overall outcomes. The SGA cohort has resulted in close to 100 published papers in peer reviewed journals and some 40 graduate and undergraduate degrees. Risk factors of SGA, like maternal smoking, low prepregnancy weight and education attainment, and a previous SGA birth outcome were confirmed. Conversely, no totally new and unknown risk factors were identified. Serial ultrasound measures have enabled a distinction between SGA with restricted and normal intrauterine growth, and has further indicated that being born SGA is mainly a problem in combination with IUGR. Further, the consequences of IUGR are more pronounced at adolescence and young adulthood than at five years of age.

An increased understanding of the pathogenesis of different categories of growth restriction is essential to recognize and diagnose IUGR properly, and to reduce the perinatal mortality and morbidity from SGA. Moreover, SGA is a significant predictor at follow-up of the child. An up to date biobank has ensured the quality of data and biological samples, and has been crucial for the outcome of the entire SGA study. It continues to be a valuable resource in future research.
\end{abstract}

This is an open access article distributed under the Creative Commons Attribution Licence, which permits unrestricted use, distribution, and reproduction in any medium, provided the original work is properly cited.

\section{INTRODUCTION}

Low birth weight and intrauterine growth restriction (IUGR) in humans are associated with a number of negative health and developmental outcomes in early life. Short-term consequences are an increased mortality and morbidity risk in the neonatal period through first year of life. Still, similar knowledge about the impact of IUGR on health in young adulthood as well as for the mothers as they approach menopause and beyond is scarce (1). Moreover, influences across generations between mother and offspring are also less well known. Cross-sectional data like low birth weight $(\mathrm{BW}<2500 \mathrm{~g})$ and small-for-gestational-age (SGA) birth, defined as a birth weight below the $10^{\text {th }}$ percentile for gestational age, gender, and parity, have both been considered adequate proxies for fetal growth restriction (1). Even so, IUGR should rather be viewed as a dynamic process caused by factors that negatively influence the growth potential of the fetus during the $2^{\text {nd }}$ and $3^{\text {rd }}$ trimester.

Well documented risk factors for SGA birth are smoking, maternal low weight or low body mass index (BMI), and a previous SGA birth (2). Increased under- 
standing of the pathogenesis of different categories of growth restriction is essential in order to recognize and diagnose IUGR properly, to reduce the perinatal mortality or morbidity, and as a predictor for a better follow-up of the child.

By use of population based data from the Norwegian Medical Birth Registry, Bakketeig et al. showed that women have a tendency to repeat gestational age and birth weight in successive pregnancies (3). Further, babies that were born similar in age and size to their elder siblings ran the lowest risk of perinatal death. For example, the perinatal mortality rate for second-birth babies between 2,501 and 3,000 g was 3.5 times higher if the first baby weighed more than $3,500 \mathrm{~g}$ instead of $3,000 \mathrm{~g}$ or less (4).

To further explore and follow up these findings, the Epidemiology and Biometry Research Program of the National Institute of Child Health and Human Development (NICHD), Department of Health Human Services, U.S.A., in 1983 issued a request for research proposals. The ensuing submissions of protocol drafts and negotiations resulted in a contract with the University of Trondheim (henceforth NTNU, The Norwegian University of Science and Technology). The contract financed the conduct of a large pregnancy and birth cohort study named the NICHD Scandinavian Successive Small-for-Gestational-Age-Births: A Longitudinal Study of Fetal Growth and Perinatal Outcome. The study was planned and organized throughout 1984-85 by NTNU and the Universities of Bergen and Uppsala as subcontractors. A similar study was conducted separately by the University of Alabama at Birmingham, U.S.A. at the same time $(5,6)$.

\section{Study aims, objectives and design}

According to the protocol approved by NICHD in 1984, the overall objective was to study all factors (medical, social, environmental, and genetic) related to fetal growth that may influence the child's physical and mental development through the first year of life (SGA I). The aim was to study different categories of IUGR births and their consequences, to identify factors that predict development during infancy, assess predictions, and to suggest preventive measures (5). Program objectives initially stated by NICHD was to identify epidemiologic risk factors among mothers who show a tendency to repeat an SGA birth in consecutive pregnancies and compare them to mothers who unexpectedly deliver such a birth (non-repeater SGA). Another aim was also to identify risk characteristics for IUGR in an attempt to distinguish between asymmetric and symmetric fetal growth retardation. The aims implied collection of prenatal medical data and interviews at regular intervals during pregnancy, including use of serial ultrasound and maternal anthropometric measures. A next aim was to evaluate postnatal growth to detect continued slow or accelerated growth during first year of life. The protocol underscored the aim to assess known (e.g., smoking, underweight, malnutrition, hypertension, and other chronic diseases in the mother) and potential new maternal risk factors prior to medical reproductive history in a population of pregnant women.

Auxiliary studies initiated by the Scandinavian researchers required collection of blood samples from the participants. One aim was to study maternal glucose tolerance and glycosylated hemoglobin related to birth weight and to assess ferritin concentration in maternal serum as an indicator of iron stores during pregnancy. Maternal serum estradiol and human placental lactogen (HPL) were studied as possible indicators of placental and feto-placental function. Nutrition status on trace elements and corresponding protein fractions in maternal and umbilical cord blood was also focused. The blood samples were at the core of the SGA biobank described later in this paper. Samples were collected for flow cytometry of the placenta, and in case of a fetal demise, a comprehensive autopsy was conducted.

A stage two of the study, Successive Small-forGestational-Age Births. The Follow-up Study of Mental, Physical, and Behavioral Development in Children Aged Five years old (SGA II) was organized as a comparison between children who were born SGA and a separate subgroup of non-SGA births. The overall objective was to assess the outcome among SGA offspring as a group. Emphasis was on the child's physical and mental health in relation to previous information from pregnancy and first year of life. The main focus was on fetal growth and its associated co-factors, and neurodevelopmental characteristics throughout childhood.

\section{Aims of this paper}

Thirty years on, we have searched the body of scientific publications from the successive SGA pregnancy and birth cohort study in an attempt to assess if and to what extent the main aims and objectives were achieved. Here, we will try to summarize the overall outcomes until and including 2014. How do the findings compare with the stated aims and objectives described in 1984, and were there any unexpected spin-off results? What is the current status of the SGA biobank and what potential - if any - does it have for future research?

\section{MATERial AND METHOD}

We have studied background documents from 1983-85 when the NICHD Scandinavian Successive SGA study was conceived, planned and organized. They include selected official communications between the local stakeholders, i.e. the universities of Bergen, Uppsala and Trondheim, and between the Faculty of Medicine, NTNU as main contractor and the US NICHD. At the core is the technical proposal, i.e. study protocol which outlined design, aims and objectives, time frames, deliverables, and ethical approvals. Moreover, there 
was a detailed business proposal covering all aspects of funding from 1984-91 when the SGA I study contract expired. We first describe the SGA study recruitment outline and procedure and the collection of clinical data and biological samples. Second, the study findings have been categorized in groups and summarized. Overall, the results have been compared with the original study objectives. Information about the biological samples (maternal and umbilical serum) was obtained through day-to-day communication with individuals who were involved in collection, organizing and storage of the samples from the onset as well as descriptions of contemporary procedures of the late 1980s. Information about data management was provided by The Regional Biobank of Central Norway, St Olavs University Hospital/NTNU. Current data on long-time storage effects on biobank serum based on quality biomarkers were assessed according to Hustad et al. (7).

\section{Recruitment and data collection in the successive SGA study}

Study recruitment took place from $1^{\text {st }}$ January 1986 through $31^{\text {st }}$ March 1988. Overall, 6,354 para 1 and para 2 Caucasian pregnant women in the three sites were referred to the study, of whom 5,722 were eligible and came to the first appointment (Figure 1). The women were divided into three groups: 1) $10 \%$ of the eligible women were randomly selected from the $5,722(\mathrm{~N}=561)$ and came to be viewed as a population reference group ("controls") of parous women in the catchment areas. 2) The study population was enriched with participants with an anticipated higher than average risk for giving birth to an SGA child. A group of 1,384 women made up the "high risk for SGA birth" group. They were characterized by one or more of the following: prior SGA birth or perinatal death, smoking around time of recruitment, maternal pre-pregnancy weight $<50 \mathrm{~kg}$, or chronic maternal disease or hypertension. 3) The remaining 3,777 women with lower than average risk for SGA represented a "Rest population" and were "put on hold" through the rest of their pregnancy. If any of them delivered a child that was classified as SGA, the birth was considered eligible and included in the detailed follow-up during the first year of life and at 5 years, even if detailed prenatal data were not available. The recruitment of participants is described in detail by Bakketeig et al. (6).

Participation included four antenatal study visits during the $2^{\text {nd }}$ and $3^{\text {rd }}$ trimester and took place around $17,25,33$ and 37 weeks of gestation. Data from ultrasound examination, maternal anthropometrics, clinical history and findings, social, medical, family, and smoking history, whole blood samples, and diet information were collected. The data collection was standardized between the three study sites (5). A few (1-3) specially trained midwives at each study site conducted all the interviews and clinical examinations

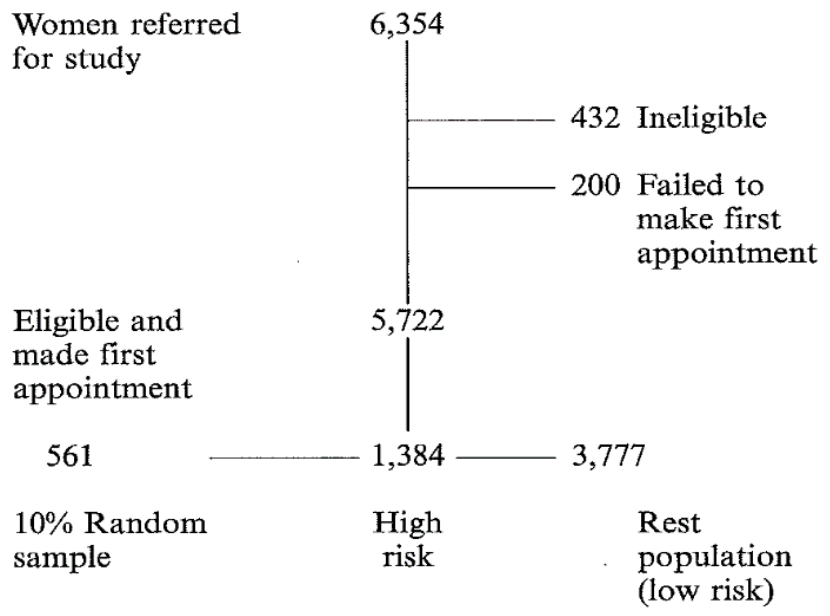

Figure 1. Flow chart illustrating the inclusion of study subjects. 6,354 para 1 and para 2 pregnant women of Caucasian origin were referred by maternity clinics, obstetricians, or family practitioners to the study. Assignment to the respective three groups took place at the university hospital during the first study interview in week $17-19$ of gestation. 561 women became the " $10 \%$ random sampled" control group, 1,384 women were assessed with risk factors for SGA outcome, called the "high risk" group. The "rest population" assessed with low risk for SGA outcome consisted of 3,777 pregnant women.

during the four study visits. That included a separate training program to improve and harmonize their skills in ultrasonography. At birth, comprehensive delivery and placenta information, including umbilical cord blood, was collected (6). A detailed pediatric examination was conducted according to a separate study protocol. Special attention and follow up was given to newborns who were admitted to the neonatal intensive care unit (NICU). Follow up during first year of life included data from the regular public health controls at 6 weeks and at 3,6, and 9 months of age. At 13 months of age, a comprehensive medical examination was conducted and included a medical history, anthropometry, questionnaires, and several neuro-motor and developmental tests.

While the SGA I study contract covered data collection during pregnancy up to 13 months of life of selected children, the three universities entered into a second, similar round of negotiations with NICHD that aimed to conduct a 5 year follow up of the same group of children (SGA study II; n=913). Those data were collected between April 1991 and March 1994. The number of invited children who were examined at 13 months and 5 years of age are shown in Table 1. Thus, $662(73 \%)$ children were examined at 13 months of age while $618(68 \%)$ children attended at 5 years. Overall, $523(57 \%)$ were seen on both occasions, of whom 225 (43\%) were classified as SGA and 298 as controls. Comparisons between SGA and non-SGA births focused on physical growth, sensory function, neuro-motor and mental function, and behavior through a battery of physiological and psychological tests. 
Table 1. Attendance of SGA births and control infants at 13 months and 5 years follow up by the three study sites. The study individuals that were examined at 5 years did not include all those who were seen at 13 months. Number of children who participated on both occasions is given in parenthesis in the far right column.

\begin{tabular}{l|cc|rcc|ccc}
\hline & \multicolumn{2}{|c|}{ At delivery } & \multicolumn{3}{c|}{13 months } & \multicolumn{3}{c}{5 years } \\
\cline { 2 - 9 } & SGA & Controls & SGA & Controls & Attendance rate (\%) & SGA & Controls & Attendance rate (\%) \\
\hline Uppsala & 197 & 270 & 130 & 149 & 60 & $105(77)$ & $139(103)$ & 52 \\
Trondheim & 101 & 121 & 86 & 98 & 83 & $82(73)$ & $104(94)$ & 83 \\
Bergen & 103 & 121 & 90 & 109 & 89 & $81(75)$ & $107(101)$ & 84 \\
\hline Total & 401 & 512 & 306 & 356 & 73 & $268(225)$ & $350(298)$ & 68 \\
\hline
\end{tabular}

\section{Biological samples from the SGA study cohort}

The blood sampling procedures differed slightly between study sites, mostly as a result of differences in clinical regimes. In Trondheim and Bergen samples were drawn four times during pregnancy, i.e. at gestational week 17, 25, 33 and 37. In Uppsala samples were collected at gestational week $17,25,33$ and at delivery. Umbilical cord blood was collected at all three sites. Not all participants succeeded to give blood samples at all five times. In Trondheim and Bergen, blood samples were collected at two or more time points from $95 \%$ of the women, in Uppsala from $88 \%$.

Phlebotomy was performed between 8 am and 4 pm on weekdays. According to the protocol, the participants were only fasting before blood sampling at 25 and 37 weeks of gestation. Collecting tubes with EDTA were used for whole blood. Collecting tubes with no additives, no silicon clot activator and no separating gel were used for serum samples. The serum sample tubes were centrifuged for 10 minutes at 4000 rpm. The time between sampling and centrifugation was 30 minutes or longer, and varied. Umbilical blood was collected in serum tubes minutes after birth and stored in a refrigerator from two hours to up to three days before centrifugation.

After centrifugation, serum was aliquoted into five NUNC tubes with glass pipettes, capped with high density polyethylene (HDPE) caps, and given labels with name and a 4-digit SGA ID-number. The excess aliquots not used for quantitation initially were stored at $-80^{\circ} \mathrm{C}$. Unfortunately, EDTA samples and blood clots that could be used for genetic analysis today were not preserved. During the next two decades most of the serum collection was stored almost untouched. Yet, some aliquots were taken out of the freezers, and some of them were refrozen.

\section{New provisions requiring reorganization of the biobank}

New data technology gradually replaced older one and by 1999 , the vast amount of study data was completely modernized and reorganized as a "Version 2.0" that enhanced the availability and data access considerably. In general, the data base held up to 2,950 clinical variables for individual mother and child pairs. A more recent overhaul of the study data (2010-14) has resulted in a "Version 3.0" that allows a seamless access to data and serum samples stored in the biobank. Behind these efforts were two official Norwegian reports;
"Biobanks. Collection, storage, use and destruction of human biologic material" (NOU 2001:19) (8), and "Good research - better health" (NOU 2005:1) (9). These reports and corresponding laws laid a pressure on the SGA biobank owners to reorganize the sample collection accordingly and in compliance with requirements from the Regional (Central Norway) Committee for Medical and Health Research Ethics (REK). That included new informed consents from the participants and secured the anonymity of the samples and data.

Since a reorganisation in 2010, the SGA serum samples have been in the custody of the biobank at The Nord-Trøndelag Health Study (The HUNT Study) (10). All samples were anonymized, thawed once, split into aliquots of $500 \mu \mathrm{l}$ in matrix tubes made of polypropylene, and capped with corks made of santoprene. Matrix tubes with individual two dimension (2D) codes on the bottom were scanned before archival at $-80^{\circ} \mathrm{C}$. The $2 \mathrm{D}$ code, the SGA study identity number of the woman, and the gestational week when the sample was drawn, have been coupled in a database at HUNT. All clinical, questionnaire, and biosample data from the SGA biobank are currently organized by The Regional Biobank of Central Norway, NTNU.

This biobank database is a customized, central Microsoft SQL-database system that communicates with a Java web application with a rich user interface (11). The database is able to handle a great diversity of sample types, analytical results, and data collected in various projects. All access to the database is protected by several layers of high-security login procedures and all actions performed in the database are recorded with a timestamp and the login identity.

The database is constructed in such a way that personal id-number is automatically encrypted using a strong encryption logarithm and it generates a unique identification number for every new sample or data unit. The key files, which contain the links between the patient identity and the encrypted number, are kept separate from the project data under strict access control. The security of the database has been formally analyzed for risk and vulnerability by the IT-department of the Central Norway Health Authority whose conclusion is that the conceivable risk is kept at a minimum (11).

\section{Research ethics}

This paper is based on previously published data from the SGA study. The women recruited to the SGA study 
signed an informed consent form before study entry. The study protocol was approved by a separate Institutional Review Board (IRB) for research involving humans in all three study sites (6). In 2010, an ethics approval for extended containment of individual participant data was obtained from Central Norway REK (REK no. 2010/1449-5). In 2014, a recent follow up questionnaire study of mothers (mean age 59 years) and offspring (mean age 27 years) was approved (2014/496/REK midt).

\section{RESULTS AND PUBLISHED FINDINGS}

Among the 561 mothers in the $10 \%$ random sample (Figure 1), 49 delivered an SGA birth (prevalence: 9\%). The corresponding number in the high risk group $(n=1,384)$ was 225 (prevalence: $16 \%)$. The remaining 127 of the 401 SGA births (Table 1) were delivered by the 3,777 mothers in the rest population (prevalence: $3.5 \%)$.

\section{The effects of smoking and other life style characteristics}

Smoking as the most prominent predictor of SGA was confirmed $(6,12-17)$. Serial ultrasound measures indicated that the growth retardation related to smoking starts after gestational week $17(18,19)$. Smoking even has a negative effect on fetal growth in mothers who stopped smoking during pregnancy (17). Maternal smoking seems to affect male fetuses more than female ones (20). High maternal pre pregnancy BMI, high weight or high weight gain during pregnancy showed no protective effect on smoke related growth retardation (17). Smoking during pregnancy may also be a risk factor for childhood overweight $(21,22)$. In addition, smoking affects the glucose homeostasis in the direction of gestational diabetes. Thus, fetal growth restriction caused by smoking seems to be stronger than the expected growth stimulation effect from higher blood glucose levels in smokers (23). Moreover, smoking is associated with lower than average score in physical and mental tests both at 13 months of age (13) as well as among adolescents (24). High caffeine intake in the third trimester may result in higher risk of fetal growth restriction, especially of the male fetus (25). IUGR is also associated with elevated homocysteine levels in maternal blood of smokers, but not non-smokers (26). Low birth weight was significantly associated with high maternal hemoglobin levels among smokers, while there was no correlation between maternal hemoglobin in late pregnancy and birth weight among non-smokers (27). Hemoglobin levels during pregnancy were significantly associated with maternal age, smoking, BMI, and iron supplementation. Except for the latter, similar associations with ferritin were not seen (28). The diet of pregnant smokers contained more fat and was less nutritious than the diet of pregnant non-smokers (29), and previous and current psychosocial conditions of pregnant women were associated with heavy smoking in the third trimester (30).

Except for the constant reminder that pregnant women should not smoke, the decrease in smoking prevalence from around $30 \%$ among parous women to below $10 \%$ at present, obviously has occurred regardless of this study (31). On the other hand, less than $5 \%$ of women reported that they drank any alcohol during the third trimester. Thus, as a means to counter the current trend that women of childbearing age drink considerably more than their peers a generation before, the SGA study has little firm documentation to offer as regards the favorable effect of alcohol abstinence (12).

\section{Other health outcomes}

SGA data have also been used to study cognitive abilities and development in children at the age of one and five years old in relation to breastfeeding and prolonged crying (32). Another study reported changes in maternal androgen in connection with nausea and vomiting during pregnancy and risk of breast cancer (33).

\section{Search for predictive markers for $S G A$}

Characteristics with properties to enhance fetal growth prediction were studied. Both maternal symphysisfundus height and fetal ultrasound biometry were significant predictors of growth retardation and SGA birth even if the variability of the latter agreed more favorably with the outcome (34-36). Dubowitz assessment of gestational age at birth agreed well with ultrasound measures at gestational week 17-20 in SGA and premature children, but not in children born after term (37). Maternal physical characteristics like BMI, weight gain during pregnancy and parental birth weight were all associated with fetal growth (38-40). Also, social and environmental factors had an impact on fetal growth $(38,39)$, while stress in personal relations, anxiety, depression, or untoward events during pregnancy did not influence birth weight $(12,41)$.

Biomarkers for more accurate prediction of an SGA birth has not been identified so far. Antiphospholipid antibodies in maternal serum in gestational week 33 did not predict SGA birth (42), while elevated testosterone levels during pregnancy was slightly associated with low birth weight (43). Human placental lactogen and estriol were positively related to fetal size, while human Chorio Gonadotropine (hCG) was not. Estriol levels were measured twice as high among mothers with the largest children (44). Still, no biomarker with unambiguous and undisputable predictive prosperities for SGA was identified $(45,46)$. The average placental cell division measured post partum by flow cytometry was slightly lower in SGA than non-SGA births, but the difference was too small to distinguish well between them (47).

\section{Morbidity and mortality among SGA births}

Being SGA was a risk factor associated with stillbirth, either before or during birth, mainly because of 
asphyxia $(48,49)$. SGA was also associated with increased neonatal morbidity. Moreover, SGA children were hospitalized more often, mainly due to respiratory tract infections (15). Such cases were more afflicted and influenced the child's health for a longer time if the mother smoked around the time of conception (15).

\section{Cognitive and motor development, and mental health of $S G A$ children}

Cognitive development, physical and mental health, and motor performances were studied at the 5 year follow up (SGA II). The differences in size between SGA and non-SGA children were evened out, and they seemed to be more related to genetic factors at 13 month of age (50). Comparisons of the IQ showed that the cognitive development in early life is strongly associated with parental factors, but only marginally associated with status as SGA or not (51). The group of SGA children were not found to be associated with preschool behavior problems (52) and they achieved the same mean test score for neuropsychological and motor functions $(53,54)$. In term SGA adolescents, minor emotional, behavioral and attention deficit symptoms were observed (55). A positive effect of breastfeeding on cognitive development in infants born SGA was indicated and the impact increased by length of breastfeeding $(32,56)$. Low socioeconomic status was the largest risk factor for developing psychiatric symptoms among SGA children (57).

\section{After nearly 30 years: Are stored biological samples of any value for future research?}

The above is a relevant question after storage of the serum samples at $-80{ }^{\circ} \mathrm{C}$ for almost three decades. Some examples of challenges and potential limitations are offered below. For instance, biomarker quantitations on 150 serum samples from the SGA biobank showed that the inverse relation between total homocysteine and folate seemed to lack in most samples. This indicates that serum has been in contact with blood cells for more than half an hour after they were drawn and before centrifugation $(7,58)$. On the other hand, the average content of homocystein in the same samples was within the reference range (mean: 6.0 $\mu \mathrm{mol} / \mathrm{L}, \quad \mathrm{SD}: 1.85 \mu \mathrm{mol} / \mathrm{L}$, reference range: $5-15$ $\mu \mathrm{mol} / \mathrm{L})$. Some of the serum samples may have been stored in room temperature beyond the recommended time since the average choline was elevated. Thus, in samples stored at room temperature phosphatidylcholine is catalyzed by phospholipase to choline at a rate of $3-5 \%$ per hour during the first hours after sampling, after which the rate increases (7). The average choline concentration in another 501 samples from the SGA biobank was $31 \mu \mathrm{mol} / \mathrm{L}$ (SD: $64.8 \mu \mathrm{mol} / \mathrm{L}$, reference range: $5-12 \mu \mathrm{mol} / \mathrm{L})$. Moreover, a large individual variation was observed. The highest average choline concentration was measured in umbilical cord blood (44 $\mu \mathrm{mol} / \mathrm{L})$, whereas the average arginine content in the latter samples was $115 \mu \mathrm{mol} / \mathrm{L}$ (SD: $35 \mu \mathrm{mol} / \mathrm{L}$, reference range $10-80 \mu \mathrm{mol} / \mathrm{L})$ after storage for 26 years.
High arginine concentrations in stored serum samples indicate proteolysis (7). This is another common limitation for long-term storage of serum samples that must be considered in later analyses of study data.

\section{Concerted action with local academics}

The Low Birth Weight (LBW) in a Life Time Perspective Study was organized and conducted at St Olavs Hospital, Trondheim and included births with BW $<1500 \mathrm{~g}(\mathrm{n}=60)$. All were born in Central Norway in 1987-89 (59). When these subjects approached puberty, two separate reference groups were recruited from the Trondheim SGA subpopulation: a) A group of term ( $>37$ weeks gestation) births with $\mathrm{BW}<10^{\text {th }}$ percentile (SGA group; $n=54$ ) and $b$ ) A group of term nonSGA births (controls; $\mathrm{n}=66$ ). Consequently, a number of the Trondheim SGA study group have been exposed to a series of detailed, comprehensive and advanced follow up procedures at age 14, 19, 23, and 26 years.

Among adolescents, one in four of the very low birth weight (VLBW) children and one in six of the SGA children had motor skill problems compared with non-SGA children. For SGA children, an increased risk of motor problems particularly concerned manual dexterity in boys (60). This group may also be at risk for visual functional problems $(61,62)$. Children born with low birth weight whether caused by preterm birth or by fetal growth retardation at term, seem to be at increased risk for psychiatric disorders as young adults (63).

Some follow up studies of VLBW children have compared findings with the SGA group. The overall conclusion is that the clinical consequences of being VLBW at 19 years are much greater than for term SGA births and include physical, mental and cognitive development in childhood and adolescence (64-66). MR imaging and cognitive tests have shown the same pattern (67-69).

\section{DiscuSSION}

SGA is a heterogeneous group and the causes for being born small are complex. A "rule of thumb" is that one third of SGA births are growth restricted, while the rest is not (70). Genetically, a fetus may be programmed to a certain size and classified as SGA without being growth restricted (71). Consequently, growth curves used in diagnostics need to adjust for the population under study, maternal parity and previous birth outcomes of the mother, and gender of the child. Since the birth weight of an SGA offspring is related to the birth weight of its parents, some women tend to repeat an SGA birth in consecutive pregnancies $(3,4)$. The risk for having an SGA birth is thus, twice as high if a previous outcome was SGA. Both genetic and environmental factors can explain this trait, but it is still hard to distinguish between them (38). Even though the growth curves for "repeater" SGA and "non-repeater" SGA mothers are almost similar, they are still grossly different from the growth curves of non-SGA births (72). 
Overall, the successive SGA study gave well founded answers to the original aims and objectives. Thus, the study confirmed the importance of a number of well documented risk factors for an SGA birth in previous epidemiologic studies. Moreover, the fiveyear follow up study systematically described the development of SGA children compared to their nonSGA peers. Ensuing sub studies through adolescence and at young adult age have confirmed the negative deviations in cognition, motor development, number of psychiatric symptoms, and educational attainment at around 20 years of age. Through the SGA study, a better understanding has been reached of how restricted intrauterine growth can be measured by use of serial ultrasound measures and how it is influenced by known maternal risk factors. Further, SGA births with signs of IUGR develop differently both in comparison with non-SGA births, but also within the SGA group without such signs. These finding will be subject to later follow up studies of the same cohort.

As expected, we have confirmed that smoking is probably the most prominent risk factor for SGA. Thus, long term follow up of offspring who were exposed to the "smoking epidemic" 1960-2000 has been advocated (73). Also, the perception of a previous SGA as a significant predictor was confirmed. On the other hand, we were only partly able to show that a "repeat" SGA is the result of hereditary characteristics or an untoward maternal lifestyle, or a combination of the two. We identified no new risk factors or biomarkers that unambiguously may predict SGA.

No firm differences between SGA pregnancies with predisposed risk and pregnancies that unexpectedly ended with an SGA birth were found, nor were any differences observed between these two groups regarding the child's development.

The initial aim to study the effect of different categories of IUGR has only partly been achieved. The majority of studies have compared SGA and non-SGA, but with less emphasis on different types of IUGR. It was an intention to distinguish between symmetric and asymmetric SGA births and anthropometric measures made it possible by use of a body mass/ponderal index. A few papers from the SGA cohort have done that, for example Vik et al. (19), but most have not.

\section{Strengths}

The overall study design was highly suitable for both cross-sectional and longitudinal studies due to the strict timing of data collection at several specified gestational ages during the second and third trimester of pregnancy, at birth, and through a series of later follow up of the participants. The large randomly selected reference group of parous women in three geographical areas gave a representative picture of the background population. The high risk group - enriched with mothers with a negative previous reproductive outcome or medical history - secured enough SGA cases for further study. The participation throughout the entire study period with $68 \%$ attendance at five years of age was quite favorable.

During pregnancy and infant follow up, all participants were followed by a few specially trained midwives and clinicians at each study site, thus minimizing inter-observer bias. In the most recent papers, serial ultrasound measurements has made it possible to identify fetal growth patterns in detail (74).

\section{Limitations}

SGA is merely a statistical concept. However, newborns who are growth restricted can be recognized by first identifying all SGA births. On the other hand, IUGR may be overlooked among appropriate for gestational age (AGA) births who are thus false negative ones. This might lead to lack of statistical power in studies where SGA and non-SGA groups are compared. It indicates that one should look for fetal growth restriction instead of SGA in follow up of pregnancies and children in future research.

The successive SGA study has not been able to identify any reliable predictive biomarker for SGA and/or IUGR so far. In 2013, Conde-Agudelo et al. did a systematic review of predictive biomarkers for IUGR (75). They concluded that none have been found so far, and that biomarkers only may be useful in combination with anthropometric and other clinical data (75). In fact, this may be a reflection of the large number and variety of factors, i.e., fetal, placental/ uterine and maternal, that to some degree cause SGA and IUGR $(1,2)$.

\section{Long term implications}

The SGA follow-up studies showed some consequences of being born SGA in a short-term perspective. Thus, childhood morbidity was higher than in the nonSGA group. Further, the SGA study has confirmed that cognitive development, physical and mental health and motor performances are closer related to genetic and socioeconomic factors than SGA per se, even though the SGA group had lower scores in physical, mental and cognitive tests. The lack of statistical power in some of these studies might be caused by the fact that the SGA group contains both IUGR and non-IUGR children. Even though differences in brain structures have been identified by MRI (76), it indicates that long term effects of SGA may be more prevalent at 15 years and higher ages, than at age one or five. They may even have some more serious implications if they are first ascertained later in life.

\section{The SGA biobank}

Storing conditions of biological material in biobanks are crucial for the quality and further use of the serum samples. If the biomaterial is not kept under stable conditions during long-term storage, a decrease in quality may have negative consequences and impose a threat to the statistical power in future studies (77). Factors like storage temperature and length of time, as well as number of thaw/freeze cycles and quality of containers all matter and should be acknowledged. The 
quality of serum samples in the current SGA biobank seems to be good, but may depend on which components that are analyzed. The potential of the SGA biobank is still high, regarding its quality and large number of samples. As a result of the comprehensive reorganization of the data and biobank (2010), data and serum samples are easily accessible and well suited for further research.

\section{CONCLUSION}

Through summary of the vast majority of published papers from the two successive SGA birth cohort studies, we have found that it has given well-founded answers to the initial research questions. A suitable study design vouches for their validity. Risk factors of SGA like maternal smoking, previous SGA outcome, and low pre pregnancy weight were confirmed. Serial ultrasound measures through second and third trimester have enabled a distinction between SGA births with and without restricted intrauterine growth. The SGA study has indicated that being born SGA is mainly a problem when it is the result of fetal growth restriction. In future SGA studies, it is paramount to distinguish between fetuses that are growth restricted or not. Studies of symmetrically as opposed to asymmetrically growth restricted SGA births were fewer than anticipated and any new and valid biomarkers to predict the condition have not been identified.

Some consequences of being born SGA were clarified through the five-year follow-up. For SGA births as a group, few and fairly small negative developmental issues stood out. As we have indicated, however, the negative consequences of fetal growth restriction as regards physical, mental and psychological development among SGA births are apparently more prevalent at 15 years of age and even beyond. This merits more detailed follow up in young adulthood and cross generational studies of mother and offspring dyads.

Large and systematic work has been undertaken to collect data through many years. A well-organized biobank ensures the quality of data and biological material for future research on the cohort. The current biobank makes the access to both data and biosamples simple and is a valuable resource when future research on the SGA cohort is considered.

\section{ACKNOWLEDGMENTS}

This study was funded by The Liaison Committee between Mid Norway Health Authority and Mid Norway Regional University Colleges, 2011 and 2012. Funding was also provided by Faculty of Medicine, NTNU Norwegian University of Science and Technology, 2008-10. We thank Kari Skjesol Torbergsen (Faculty of Technology, NTNU) for her kind facilitation and contribution to this project.

\section{REFERENCES}

1. Mandy GT. Small for gestational age. UpToDate, Norwegian health library, 2014.

2. McCowan L, Horgan RP. Risk factors for small for gestational age infants. Best Pract Res Clin Obstet Gynaecol 2009;23(6):779-93.

3. Bakketeig LS, Hoffman HJ, Harley EE. The tendency to repeat gestational age and birth weight in successive births. Am J Obstet Gynecol 1979;135(8):1086-103.

4. Bakketeig LS, Hoffman HJ. The tendency to repeat gestational age and birth weight in successive births, related to perinatal survival. Acta Obstet Gynecol Scand 1983;62(5):385-92.

5. Bergsjo P, Hoffman HJ, Davis RO, et al. Preliminary results from the Collaborative Alabama and Scandinavian Study of Successive Small-for-Gestational Age Births. Acta Obstet Gynecol Scand 1989;68(1):19-25.

6. Bakketeig LS, Jacobsen G, Hoffman HJ, et al. Pre-pregnancy risk factors of small-for-gestational age births among parous women in Scandinavia. Acta Obstet Gynecol Scand 1993;72(4):273-9.

7. Hustad S, Eussen S, Midttun O, et al. Kinetic modeling of storage effects on biomarkers related to B vitamin status and one-carbon metabolism. Clin Chem 2012;58(2):402-10.

8. NOU 2001:19 Biobanker. Innhenting, oppbevaring, bruk og destruksjon av humant biologisk materiale. Oslo: Sosial-og helsedepartementet, 2001.

9. NOU 2005:1 God forskning - bedre helse, Helseforskningsloven. Oslo: Helse- og omsorgsdepartementet, 2005.

10. The Nord-Trøndelag Health Study. http://www.ntnu.edu/hunt.

11. Halgunset J, Mikkelsen ØL, Skogseth H. The Regional Research Biobank of Central Norway - "One biobank, many collections". Norsk Epidemiologi 2012;21(2):177-84.

12. Jacobsen G, Schei B, Hoffman HJ. Psychosocial factors and small-for-gestational-age infants among parous Scandinavian women. Acta Obstet Gynecol Scand Suppl 1997;165:14-8.

13. Trasti N, Vik T, Jacobsen G, Bakketeig LS. Smoking in pregnancy and children's mental and motor development at age 1 and 5 years. Early Hum Dev 1999;55(2):137-47.

14. Vik T, Jacobsen G, Vatten L, et al. Pre- and post-natal growth in children of women who smoked in pregnancy. Early Hum Dev 1996;45(3):245-55.

15. Vik T, Vatten L, Markestad T, et al. Morbidity during the first year of life in small for gestational age infants. Arch Dis Child Fetal Neonatal Ed 1996;75(1):F33-7. 
16. Zaren B, Lindmark G, Gebre-Medhin M. Maternal smoking and body composition of the newborn. Acta Paediatr 1996;85(2):213-9.

17. Zaren B, Cnattingius S, Lindmark G. Fetal growth impairment from smoking - is it influenced by maternal anthropometry? Acta Obstet Gynecol Scand Suppl 1997;165:30-4.

18. Bergsjo P, Bakketeig LS, Lindmark G. Maternal smoking does not affect fetal size as measured in the midsecond trimester. Acta Obstet Gynecol Scand 2007;86(2):156-60.

19. Vik T, Vatten L, Jacobsen G, et al. Prenatal growth in symmetric and asymmetric small-for-gestational-age infants. Early Hum Dev 1997;48(1-2):167-76.

20. Zaren B, Lindmark G, Bakketeig L. Maternal smoking affects fetal growth more in the male fetus. Paediatr Perinat Epidemiol 2000;14(2):118-26.

21. Ruckinger S, Beyerlein A, Jacobsen G, et al. Growth in utero and body mass index at age 5 years in children of smoking and non-smoking mothers. Early Hum Dev 2010;86(12):773-7.

22. Wideroe M, Vik T, Jacobsen G, et al. Does maternal smoking during pregnancy cause childhood overweight? Paediatr Perinat Epidemiol 2003;17(2):171-9.

23. Zaren B, Lindmark G, Wibell L, et al. The effect of smoking on glucose homeostasis and fetal growth in pregnant women. Upsala J Med Sci 2000;105(1):41-56.

24. Indredavik MS, Brubakk AM, Romundstad $P$, et al. Prenatal smoking exposure and psychiatric symptoms in adolescence. Acta Paediatr 2007;96(3):377-82.

25. Vik T, Bakketeig LS, Trygg KU, et al. High caffeine consumption in the third trimester of pregnancy: genderspecific effects on fetal growth. Paediatr Perinat Epidemiol 2003;17(4):324-31.

26. Carlsen SM, Jacobsen G, Vatten L, et al. In pregnant women who smoke, caffeine consumption is associated with an increased level of homocysteine. Acta Obstet Gynecol Scand 2005;84(11):1049-54.

27. Zaren B, Lindmark G, Bergsjo P. Hemoconcentration in smoking mothers is associated with impaired fetal growth. Acta Obstet Gynecol Scand 1997;76(10):933-41.

28. Rasmussen S, Bergsjo P, Jacobsen G, et al. Haemoglobin and serum ferritin in pregnancy - correlation with smoking and body mass index. Eur J Obstet Gynecol Reprod Biol 2005;123(1):27-34.

29. Trygg K, Lund-Larsen K, Sandstad B, et al. Do pregnant smokers eat differently from pregnant non-smokers? Paediatr Perinat Epidemiol 1995;9(3):307-19.

30. Thue E, Schei B, Jacobsen G. Psychosocial factors and heavy smoking during pregnancy among parous Scandinavian women. Scand J Prim Health Care 1995;13(3):182-7.

31. Oien T, Storro O, Jenssen JA, et al. The impact of a minimal smoking cessation intervention for pregnant women and their partners on perinatal smoking behaviour in primary health care: a real-life controlled study. BMC Public Health 2008;8:325.

32. Angelsen NK, Vik T, Jacobsen G, et al. Breast feeding and cognitive development at age 1 and 5 years. Arch Dis Child 2001;85(3):183-8.

33. Carlsen SM, Vanky E, Jacobsen G. Nausea and vomiting associate with increasing maternal androgen levels in otherwise uncomplicated pregnancies. Acta Obstet Gynecol Scand 2003;82(3):225-8.

34. Jacobsen G. Prediction of fetal growth deviations by use of symphysis-fundus height measurements. Int $J$ Technol Assess Health Care 1992;8 Suppl 1:152-9.

35. Jacobsen G. Detection of intrauterine growth deviation. A comparison between symphysis-fundus height and ultrasonic measurements. Int J Technol Assess Health Care 1992;8 Suppl 1:170-5.

36. Wikstrom I, Bergstrom R, Bakketeig L, et al. Prediction of high birthweight from maternal characteristics, symphysis fundal height and ultrasound biometry. Gynecol Obstet Invest 1993;35(1):27-33.

37. Vik T, Vatten L, Markestad T, et al. Dubowitz assessment of gestational age and agreement with prenatal methods. Am J Perinatol 1997;14(6):369-73.

38. Magnus P, Bakketeig LS, Hoffman H. Birth weight of relatives by maternal tendency to repeat small-forgestational-age (SGA) births in successive pregnancies. Acta Obstet Gynecol Scand Suppl 1997;165:35-8.

39. Nodtvedt AM, Jacobsen G, Balstad P, et al. [Social class and birth weight]. Tidsskr Nor Laegeforen 1999; 119(30):4455-9.

40. Shepard MJ, Bakketeig LS, Jacobsen G, et al. Maternal body mass, proportional weight gain, and fetal growth in parous women. Paediatr Perinat Epidemiol 1996;10(2):207-19.

41. Rostad B, Schei B, Jacobsen G. Health consequences of severe life events for pregnancy. Scand J Prim Health Care 1995;13(2):99-104.

42. Schei B, Ostensen M, Moen T, Jacobsen G, et al. Can maternal antiphospholipid antibodies predict the birth of a small-for-gestational-age child? Acta Obstet Gynecol Scand 1995;74(6):425-8.

43. Carlsen SM, Jacobsen G, Romundstad P. Maternal testosterone levels during pregnancy are associated with offspring size at birth. Eur J Endocrinol 2006;155(2):365-70.

44. Kaijser M, Granath F, Jacobsen G, et al. Maternal pregnancy estriol levels in relation to anamnestic and fetal anthropometric data. Epidemiology 2000;11(3):315-9.

45. Markestad T, Bergsjo P, Aakvaag A, et al. Prediction of fetal growth based on maternal serum concentrations of human chorionic gonadotropin, human placental lactogen and estriol. Acta Obstet Gynecol Scand Suppl 1997; 165:50-5.

46. Kaijser M, Jacobsen G, Granath F, et al. Maternal age, anthropometrics and pregnancy oestriol. Paediatr Perinat Epidemiol 2002;16(2):149-53. 
47. Markestad T, Lossius P, Maartmann-Moe H, et al. Cell division in placentas of appropriate and small-forgestational-age infants. A flow cytometry study. Acta Obstet Gynecol Scand Suppl 1997;165:59-62.

48. Isaksen CV, Laurini RN, Jacobsen G. Pre-pregnancy risk factors of small-for-gestational-age births and perinatal mortality. Acta Obstet Gynecol Scand Suppl 1997;165:44-9.

49. Vik T, Markestad T, Ahlsten G, et al. Body proportions and early neonatal morbidity in small-for-gestationalage infants of successive births. Acta Obstet Gynecol Scand Suppl 1997;165:76-81.

50. Markestad T, Vik T, Ahlsten G, et al. Small-for-gestational-age (SGA) infants born at term: growth and development during the first year of life. Acta Obstet Gynecol Scand Suppl 1997;165:93-101.

51. Sommerfelt K, Andersson HW, Sonnander K, et al. Cognitive development of term small for gestational age children at five years of age. Arch Dis Child 2000;83(1):25-30.

52. Sommerfelt K, Andersson HW, Sonnander K, et al. Behavior in term, small for gestational age preschoolers. Early Hum Dev 2001;65(2):107-21.

53. Sommerfelt K, Sonnander K, Skranes J, et al. Neuropsychologic and motor function in small-for-gestation preschoolers. Pediatr Neurol 2002;26(3):186-91.

54. Kulseng S, Jennekens-Schinkel A, Naess P, Romundstad P, et al. Very-low-birthweight and term small-forgestational-age adolescents: attention revisited. Acta Paediatr 2006;95(2):224-30.

55. Indredavik MS, Vik T, Heyerdahl S, et al. Psychiatric symptoms and disorders in adolescents with low birth weight. Arch Dis Child Fetal Neonatal Ed 2004;89(5):F445-50.

56. Rao MR, Hediger ML, Levine RJ, et al. Effect of breastfeeding on cognitive development of infants born small for gestational age. Acta Paediatr 2002;91(3):267-74.

57. Indredavik MS, Vik T, Evensen KA, et al. Perinatal risk and psychiatric outcome in adolescents born preterm with very low birth weight or term small for gestational age. J Dev Behav Pediatr 2010;31(4):286-94.

58. Fiskerstrand T, Refsum H, Kvalheim G, et al. Homocysteine and other thiols in plasma and urine: automated determination and sample stability. Clin Chem 1993;39(2):263-71.

59. Skranes JS, Martinussen M, Smevik O, et al. Cerebral MRI findings in very-low-birth-weight and small-forgestational-age children at 15 years of age. Pediatr Radiol 2005;35(8):758-65.

60. Evensen KA, Vik T, Helbostad J, et al. Motor skills in adolescents with low birth weight. Arch Dis Child Fetal Neonatal Ed 2004;89(5):F451-5.

61. Lindqvist S, Vik T, Indredavik MS, et al. Eye movements and binocular function in low birthweight teenagers. Acta Ophthalmol 2008;86(3):265-74.

62. Lindqvist S, Vik T, Indredavik MS, et al. Visual acuity, contrast sensitivity, peripheral vision and refraction in low birthweight teenagers. Acta Ophthalmol Scand 2007;85(2):157-64.

63. Lund LK, Vik T, Skranes J, et al. Psychiatric morbidity in two low birth weight groups assessed by diagnostic interview in young adulthood. Acta Paediatr 2011;100(4):598-604.

64. Evensen KA, Lindqvist S, Indredavik MS, et al. Do visual impairments affect risk of motor problems in preterm and term low birth weight adolescents? Eur J Paediatr Neurol 2009;13(1):47-56.

65. Evensen KA, Sigmundsson H, Romundstad P, et al. Inter- and intra-modal matching in very low birth weight and small for gestational age adolescents. Early Hum Dev 2007;83(1):19-27.

66. Evensen KA, Steinshamn S, Tjonna AE, et al. Effects of preterm birth and fetal growth retardation on cardiovascular risk factors in young adulthood. Early Hum Dev 2009;85(4):239-45.

67. Indredavik MS, Vik T, Heyerdahl S, et al. Psychiatric symptoms in low birth weight adolescents, assessed by screening questionnaires. Eur Child Adolesc Psychiatry 2005;14(4):226-36.

68. Indredavik MS, Vik T, Heyerdahl S, et al. Low-birthweight adolescents: quality of life and parent-child relations. Acta Paediatr 2005;94(9):1295-302.

69. Vangberg TR, Skranes J, Dale AM, et al. Changes in white matter diffusion anisotropy in adolescents born prematurely. NeuroImage 2006;32(4):1538-48.

70. Small-for-Gestational-Age Fetus, Investigation and Management (Green-top Guideline No. 31, 2nd Edition). London, Royal College of Obstetricians and Gynaecologists, 2013.

71. Bakketeig LS. Current growth standards, definitions, diagnosis and classification of fetal growth retardation. Eur J Clin Nutr 1998;52 (Suppl 1):S1-4.

72. Bakketeig LS, Hoffman HJ, Jacobsen G, et al. Intrauterine growth pattern by the tendency to repeat small-forgestational-age births in successive pregnancies. Acta Obstet Gynecol Scand Suppl 1997;165:3-7.

73. Olsen J. David Barker (1938-2013) - a giant in reproductive epidemiology. Acta Obstet Gynecol Scand 2014; 93(11):1077-80.

74. Lohaugen GC, Ostgard HF, Andreassen S, et al. Small for gestational age and intrauterine growth restriction decreases cognitive function in young adults. $J$ Pediatr 2013;163(2):447-53.

75. Conde-Agudelo A, Papageorghiou AT, Kennedy SH, et al. Novel biomarkers for predicting intrauterine growth restriction: a systematic review and meta-analysis. BJOG 2013;120(6):681-94.

76. Martinussen M, Fischl B, Larsson HB, et al. Cerebral cortex thickness in 15-year-old adolescents with low birth weight measured by an automated MRI-based method. Brain 2005;128(Pt 11):2588-96.

77. Balasubramanian R, Muller L, Kugler K, et al. The impact of storage effects in biobanks on biomarker discovery in systems biology studies. Biomarkers 2010;15(8):677-83. 